\title{
Fuzzy Logic Strategy for Solving an Optimal Control Problem of Glucose and Insulin in Diabetic Human
}

\author{
Jean Marie Ntaganda \\ Department of Applied Mathematics, School of Pure and Applied Science, College of Science and Technology, \\ University of Rwanda (Huye Campus), Butare, Rwanda \\ Email: jmnta@yahoo.fr,jmntaganda@nur.ac.rw
}

Received August 17, 2013; revised September 27, 2013; accepted October 7, 2013

Copyright (C) 2013 Jean Marie Ntaganda. This is an open access article distributed under the Creative Commons Attribution License, which permits unrestricted use, distribution, and reproduction in any medium, provided the original work is properly cited.

\begin{abstract}
This paper aims at the development of an approach integrating the fuzzy logic strategy for a glucose and insulin in diabetic human optimal control problem. To test the efficiency of this strategy, the author proposes a numerical comparison with the indirect method. The results are in good agreement with experimental data.
\end{abstract}

Keywords: Fuzzy Logic; Optimal Control; Membership Function; Membership Degree; Glucose; Insulin; Numerical Simulation

\section{Introduction}

The blood glucose in human body is controlled by two key organs: the pancreas and the liver. The key hormones are insulin and glucagon. In the pancreas, there are clusters of endocrine cells scattered throughout the tissue. These are the $\alpha$-cells and the $\beta$-cells. The $\alpha$-cells produce glucagon and the $\beta$-cells produce insulin. The pancreas secretes these antagonistic hormones into the extracellular fluid, which then enters the circulatory system and regulates the concentration of blood glucose. For biologists, this is known as a simple endocrine pathway. Human bodies need to maintain glucose concentration level in a narrow range $70-120 \mathrm{mg} / \mathrm{dl}$. If glucose concentration level is significantly out of the normal range, this person is considered to have the plasma glucose problem: Hyperglycemia (greater than $140 \mathrm{mg} / \mathrm{dl}$ after an Oral Glucose Tolerance Test, or greater than $100 \mathrm{mg} / \mathrm{dl}$ after a Fasting Glucose Tolerance Test) or hypoglycemia (less than $40 \mathrm{mg} / \mathrm{dl})$.

Diabetes mellitus is an endocrine disorder caused by a deficiency of insulin (Type 1 Diabetes) or a decreased response to insulin in target tissues (Type 2 Diabetes) [1]. The major long-term effects of diabetes are caused by hyperglycemia. Prolonged hyperglycemia can cause complications, which may lead to kidney disease, blindness, loss of limbs, and so on. The hypoglycemia can lead to dizziness, coma, or even death. Type 1 diabetes was previously called insulin-dependent diabetes mellitus (IDDM) or juvenile-onset diabetes. It is an autoimmune disorder in which the immune system destroys the $\beta$-cells of the pancreas. Previously known as non-insulin dependent diabetes (NIDDM) or maturity onset diabetes, Type 2 diabetes is essentially a disorder of middle age onwards. However, with the increase in childhood obesity in recent years there have been increasing reports of this form of diabetes in much younger age groups. Therein lies a clue to the origin of this disorder, because Type 2 diabetes is closely linked to obesity. As the rates of obesity have soared over the last twenty years, so in tandem has the incidence of Type 2 diabetes. The underlying factor leading to Type 2 diabetes is a failure of the pancreas to produce sufficient insulin. This may be for one or both of two reasons. The first is that there are insufficient insulin producing cells in the pancreas to meet demands. The second is that there is resistance by the body's target cells to the insulin which is produced, thus requiring increasing amounts to ensure effect. Either way the ultimate poverty of effect of insulin is related to over weight or obesity. One of the main actions of insulin is to help regulate blood sugar (or to be precise, blood glucose) levels. It achieves this in part by promoting uptake of sugar by cells, that is, muscle cells. Insufficient action of insulin leads to a reduction in sugar uptake and therefore 
causes an abnormal rise in blood sugar. The result of this is the symptoms of diabetes (excessive thirst, passing of excessive amounts of urine, fatigue, frequent skin infections, e.g., boils, etc,). Type 2 diabetes often runs in families but even so the ways the patient can prevent its onset are: keep to a healthy weight, eat a healthy diet and ensure adequate levels of daily exercise. With regard to diabetes, regular physical activity aids weight management, improves insulin sensitivity and therefore blood glucose control, aids blood fats control, and improves your overall sense of well-being.

Since the 1960s, mathematical models have been used to describe glucose-insulin dynamics [2]. Bergman et al. [3] proposed a three-compartment minimal model to analyze the glucose disappearance and insulin sensitivity during an intravenous glucose tolerance test. Modifications have been made to the original minimal model to incorporate various physiological effects of glucose and insulin. Cobelli and co-workers [4] developed a revised minimal model in order to separate the effects of glucose production from utilization. The overestimation of glucose effectiveness and the underestimation of insulin sensitivity by the minimal model were addressed in yet another publication by Cobelli et al. [5] where a second no accessible glucose compartment was added to the original model. Hovorka and co-workers [6] extended the original minimal model by adding three glucose and insulin sub-compartments in order to capture absorption, distribution, and disposal dynamics, respectively. Anirban Roy et al. presented a three compartmental model to capture the changes in glucose and insulin dynamics due to exercise [7]. This model incorporates the effects of physiological exercise into the Bergman minimal model [3] in order to capture the plasma glucose and insulin dynamics during, as well as after, periods of mild-tomoderate exercise.

In this paper we are interested in the role of physical activity, and how it plays a crucial role in controlling plasma glucose level and increasing insulin sensitivity in Type 2 diabetes is highlighted through a bicompartmental model such that the controls are those of cardiovascular-respiratory system. Therefore, the formulation of optimal control problem is done. There are numerous methods that allow solving this kind of problem. We prefer to make a comparative study of direct method with another approach based on the fuzzy logic strategy.

This paper is organised as follows. Section 2 presents the model equations and optimal control problem. A short description of strategy approach by fuzzy logic for solving optimal control problems is discussed in this section. The Section 3 is interested in the application of the direct approach and the approach integrating the fuzzy logic for solving an optimal control problem of glucose-insulin in diabetic human. The numerical simulation is presented in
Section 4. Finally, we present conclusion remarks in Section 5.

\section{Methods}

\subsection{Setting of the Problem}

Taking account of the physiological properties of glucose-insulin system, we propose a model elaborated in [8] where we consider a two compartmental model composed of the liver compartment (LC) and the pancreas compartment (PC). The diagram is shown in the Figure 1.

It is well known the arterial pressure $\left(P_{A}\right)$ leads the tissues to receive the blood from cardiovascular respiratory system whereas the blood comes to cardiovascular respiratory system from tissues due to arterial pressure $\left(P_{V}\right)$. The cardiac rhythm $(H)$ and the alveolar ventilation $\left(\dot{V}_{A}\right)$ are the parameters that influence the cardiovascular-respiratory system to control these pressures. During the physical effort, the cardiac rhythm is adjusted via the baroreceptor controls while alveolar ventilation is adjusted by the respiratory control. The respiratory control system varies the ventilation rate in response to the levels of dioxide $\mathrm{CO}_{2}$ and oxygen $\mathrm{O}_{2}$ gases. Consequently, it arises the ventilation rate and cardiac output influence mutually. It is then obvious that exchanges between LC and PC are controlled by heart rate $(H)$ and alveolar ventilation $\left(\dot{V}_{A}\right)$ functions. The mechanism of this control is not direct and can be represented by outflow functions between systemic arterial and venous compartments that depend on heart rate alveolar ventilation (Figure 1).

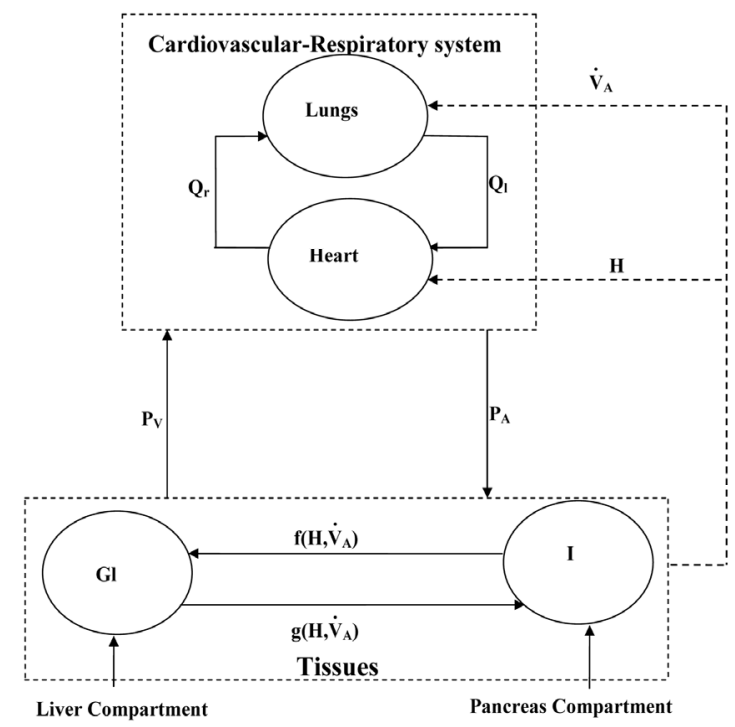

Figure 1. A schematic diagram of two compartments for modeling human glucose-insulin. $Q_{I}$ and $Q_{r}$ are left and right cardiac flow respectively. $H$ is heart rate and $\dot{V}_{A}$ denotes alveolar ventilation. $P_{A}$ and $P_{V}$ represent arterial and venous pressure respectively. 
These functions represent the mass transfer between these compartments where the exchanges are represented by the arrows in the Figure 1. Let us consider that the parameters $H$ and $\dot{V}_{A}$ reach their equilibrium values respectively $H^{e}$ and $\dot{V}_{A}^{e}$, the optimal control problem during a physical activity can be formulated as follows.

Find $H^{*}, \dot{V}_{A}^{*}$ solution of

$$
\begin{aligned}
& \min _{H, \dot{V}_{A}} J\left(H, \dot{V}_{A}\right) \\
& =\int_{0}^{T_{\max }}\left[a_{1}\left(G(t)-G^{e}\right)^{2}+a_{2}\left(I(t)-I^{e}\right)^{2}\right. \\
& \left.\quad+b_{1}\left(H(t)-H^{e}\right)^{2}+b_{2}\left(\dot{V}_{A}(t)-\dot{V}_{A}^{e}\right)^{2}\right] \mathrm{d} t
\end{aligned}
$$

subject to

$$
\begin{cases}\frac{\mathrm{d}}{\mathrm{d} t} G=-G+(I)^{(-1.3285)} f\left(H, \dot{V}_{A}\right), & G(0)=130 \\ \frac{\mathrm{d}}{\mathrm{d} t} I=-I+(G)^{(-0.6253)} g\left(H, \dot{V}_{A}\right), & I(0)=25,\end{cases}
$$

where

$$
f\left(H, \dot{V}_{A}\right) \approx \dot{V}_{A}^{0.0346}+0.7604 \dot{V}_{A} \times H+H^{0.7329}+0.8627
$$

and

$$
g\left(H, \dot{V}_{A}\right) \approx \dot{V}_{A}^{0.3011} H+0.0191 \dot{V}_{A} \times H-178.9206 H^{0.0262}
$$

with $a_{1}, a_{2}, b_{1}$ and $b_{2}$ the real constants. The variables of the mathematical model are glucose $(G)$ and insulin $(I)$.

\subsection{Description of Fuzzy Logic Strategy Approach}

Let us consider the following problem.

Find, $U^{*}=\left(U_{0}, \cdots, U_{N-1}\right)^{T} \in \mathbb{R}^{N}$, that minimizes

$$
J\left(U_{0}, \cdots, U_{N-1}\right)=\sum_{k=0}^{N-1}\left(x_{k}^{\mathrm{T}} R x_{k}+U_{k}^{\mathrm{T}} Q U_{k}\right)
$$

subject to

$$
\left\{\begin{array}{l}
x_{k+1}=f_{k}\left(x_{k}, U_{k}\right) \\
x_{k} \in \mathbb{R}, U_{k} \in \mathbb{R}
\end{array}, \quad k=0, \cdots, N-1\right.
$$

where $R$ and $Q$ are positive defined matrices.

The problem (5)-(6) can be solved by the dynamic programming method. This method has a fast convergence, its convergence rate is quadratic and the optimal solution is often represented as a state of control feedback [9]. However, the solution determined by this method depends on the choice of the initial trajectory and in some cases this solution is not optimal. It is for this reason that the integration of the fuzzy logic [10] can permit to determine quickly the optimal solution. We develop a linearization strategy of the subject system by an ap- proach based on the fuzzy logic. This approach had been developed by Takagi-Sugeno $[11,12]$. The model that has been introduced in 1985 by Takagi-Sugeno permits to get some fuzzy linearization regions in the state space [13]. While taking these fuzzy regions as basis, non linear system is decomposed in a structure multi models which is composed of several independent linear models [14]. The linearization is made around an operating point contained in these regions.

Let's consider the set of operating point $X_{i}$, $i=1, \cdots, S$. Different fuzzy approximations of the nonlinear term $N L(x)$ can be considered.

1) The approximation of order zero gives:

$$
N L(x) \approx N L_{0}(x)=N L\left(x_{i}\right)
$$

2) Using the first order of Taylor expansion series we obtain:

$$
N L(x) \approx N L_{1}(x)=N L\left(x_{i}\right)+\left(\frac{\mathrm{d} N L(x)}{\mathrm{d} x}\right)_{x_{i}}^{\mathrm{T}}\left(x-x_{i}\right) .
$$

To improve this approximation, we introduce the factor of the consequence for fuzzy Takagi-Sugeno system. This factor permits to minimize the error between the non linear function and the fuzzy approximation. If $\epsilon$ designates this factor, the approximation (8) can be formulated as the following form:

$$
\begin{aligned}
N L(x) & \approx(1-\beta) N L_{0}(x)+\epsilon N L_{1}(x) \\
& \approx N L\left(x_{i}\right)+\beta\left(\frac{\mathrm{d} N L(X)}{\mathrm{d} x}\right)_{x_{i}}^{\mathrm{T}}\left(x-x_{i}\right),
\end{aligned}
$$

with $0 \leq \beta \leq 1$.

If one replaces the term $N L$ by its value approached in (6), the linearization around $x_{i}$ leads to

$$
x_{k+1}=A_{i, k} x_{k}+B_{i, k} U_{k}+C_{i, k}, i=1, \cdots, S ; k=0, \cdots, N-1
$$

where $A_{i, k}$ and $B_{i, k}$ are square matrix which has $N \times$ $N$ order and $C_{i, k}$ matrix with $N \times 1$ order.

Therefore, the optimal control problem (5)-(6) becomes a linear quadratic problem which the feedback control is given by the following expression $[15,16]$ :

$$
U_{i, k}=-K_{i} x_{k}, i=1, \cdots, S ; k=0, \cdots, N-1,
$$

where

$$
K_{i}=\left(Q+B_{i}^{\mathrm{T}} E_{i} B_{i}\right)^{-1} B_{i}^{\mathrm{T}} E_{i} A_{i},
$$

is the feedback gain matrix and $E_{i}$ discreet Riccati equation solution of the following form

$$
E_{i}-Q-A_{i}^{\mathrm{T}} E_{i} A_{i}+A_{i}^{\mathrm{T}} E_{i} B_{i}\left(R+B_{i}^{\mathrm{T}} E_{i} B_{i}\right)^{-1} B_{i}^{\mathrm{T}} E_{i} A_{i}=0 .
$$


It is obvious that the linearization around every operating point gives the system for which the equations have the form (10). Because there are $S$ operating points, we have $S$ systems which have this form. Therefore, according to the relation (11) $S$ controls are determined. The defuzzyfication method [12] permits to determine only one system and only one control $U_{k}$.

Then, this transformation gives the following equation:

$$
\begin{gathered}
x_{k+1}=A x_{k}+B U_{k}+C, \quad k=0, \cdots, N-1, \\
U_{k}=-K x_{k}, k=0, \cdots, N,
\end{gathered}
$$

where

$$
\begin{gathered}
A=\frac{\sum_{i=1}^{S} \omega_{i}\left(x_{i}\right) A_{i, k}}{\sum_{i=1}^{S} \omega_{i}\left(x_{i}\right)}, \quad B=\frac{\sum_{i=1}^{S} \omega_{i}\left(x_{i}\right) B_{i, k}}{\sum_{i=1}^{S} \omega_{i}\left(x_{i}\right)} \\
C=\frac{\sum_{i=1}^{S} \omega_{i}\left(x_{i}\right) C_{i, k}}{\sum_{i=1}^{S} \omega_{i}\left(x_{i}\right)}, \text { and } K=\frac{\sum_{i=1}^{S} \omega_{i}\left(x_{i}\right) K_{i, k}}{\sum_{i=1}^{S} \omega_{i}\left(x_{i}\right)},
\end{gathered}
$$

and where $\omega_{i}\left(x_{i}\right)$ designates membership degree partner to the operating point $x_{i}$.

\section{Numerical Approaches for Solving the Optimal Control Problem (1)-(2)}

\subsection{Fuzzy Logic Strategy}

To approximate the optimal control problem (1)-(2), we propose to use the explicit Euler scheme. The stability of this scheme constitutes an advantage to approach some ordinary differential equations.

From the function $f$ and $g$ given by (3) and (4) respectively, the Taylor expansion around $H^{e}$ and $\dot{V}_{A}^{e}$ allows the system (2) to become

$$
\left\{\begin{array}{l}
\frac{\mathrm{d}}{\mathrm{d} t} G=-G+(I)^{(-1.3285)}\left(C_{1 f} H+C_{2 f} \dot{V}_{A}+C_{3 f}\right) \\
\frac{\mathrm{d}}{\mathrm{d} t} I=-I+(G)^{(-0.6253)}\left(C_{1 g} H+C_{2 g} \dot{V}_{A}+C_{3 g}\right),
\end{array}\right.
$$

where

$$
\begin{aligned}
C_{1 f}= & 0.7604 \times \dot{V}_{A}^{e}+0.7329\left(H^{e}\right)^{-0.2671} \\
C_{2 f}= & 0.0346 \times\left(\dot{V}_{A}^{e}\right)^{-0.9654}+0.7604 \times H^{e} \\
C_{3 f}= & f\left(H^{e}, \dot{V}_{A}^{e}\right)-1.4933 \times H^{e} \times \dot{V}_{A}^{e}-0.7329 \times\left(H^{e}\right)^{0.7329} \\
& -0.0346 \times\left(\dot{V}_{A}^{e}\right)^{-0.0346} \\
C_{1 g}= & 0.0191 \times \dot{V}_{A}^{e}-4.6877 \times\left(H^{e}\right)^{-0.9738} \\
C_{2 g}= & 0.3011 \times\left(\dot{V}_{A}^{e}\right)^{-0.6989} \times H^{e}+0.0191 \times H^{e} \\
C_{3 g}= & g\left(H^{e}, \dot{V}_{A}^{e}\right)-4.6877 \times\left(H^{e}\right)^{0.0262} \\
& -0.3011 \times\left(\dot{V}_{A}^{e}\right)^{0.3011}-0.0382 \times H^{e} \times \dot{V}_{A}^{e} .
\end{aligned}
$$

Let us set the following variable change

$X=\left(G-G^{e}, I-I^{e}\right)^{\mathrm{T}}$ and $U=\left(H-H^{e}, \dot{V}_{A}-\dot{V}_{A}^{e}\right)^{\mathrm{T}}$

Therefore, the system (17) is written as the follows

$$
\left\{\begin{aligned}
\frac{\mathrm{d} X_{1}}{\mathrm{~d} t}= & -X_{1}-G^{e}+\left(X_{2}+I^{e}\right)^{(-1.3285)} \\
& \cdot\left(C_{1 f}\left(U_{1}+H^{e}\right)+C_{2 f}\left(U_{2}+\dot{V}_{A}^{e}\right)+C_{3 f}\right) \\
\frac{\mathrm{d} X_{2}}{\mathrm{~d} t}= & -X_{2}-I^{e}+\left(X_{1}+G^{e}\right)^{(-0.6253)} \\
& \cdot\left(C_{1 g}\left(U_{1}+H^{e}\right)+C_{2 g}\left(U_{2}+\dot{V}_{A}^{e}\right)+C_{3 g}\right) .
\end{aligned}\right.
$$

Using explicit Euler scheme on an uniform grid $\Omega_{N}$, the system (19) is approximated by the following (see Equation (20))

where $h=\frac{T_{\max }}{N}$.

To approximate the objective function of the problem (1), we use the rectangular method. Hence, we obtain

$$
J^{N}(X, U)=\sum_{k=0}^{N-1}\left(X_{k}^{\mathrm{T}} R_{1} X_{k}+U_{k}^{\mathrm{T}} R_{2} U_{k}\right) h,
$$

where $X_{k}=\left(X_{1, k}, X_{2, k}\right)^{\mathrm{T}} \cdot R_{1}$ and $R_{2}$ are the matrix

$$
R_{1}=\left(\begin{array}{cc}
a_{1} & 0 \\
0 & a_{2}
\end{array}\right), \quad R_{2}=\left(\begin{array}{cc}
b_{1} & 0 \\
0 & b_{2}
\end{array}\right)
$$

The system (20) has two following nonlinear factors:

$$
\left\{\begin{aligned}
X_{1, k+1}= & (1-h) X_{1, k}+h\left(X_{2, k}+I^{e}\right)^{(-1.3285)}\left[C_{1 f} \times U_{1, k}+C_{2 f} \times U_{2, k}\right] \\
& +h\left[-G^{e}+\left(X_{2, k}+I^{e}\right)^{(-1.3285)}\left(C_{1 f} H^{e}+C_{2 f} \dot{V}_{A}^{e}+C_{3 f}\right)\right] \\
X_{2, k+1}= & (1-h) X_{2, k}+h\left(X_{1, k}+G^{e}\right)^{(-0.6253)}\left[C_{1 g} U_{1, k}+C_{2 g} U_{2, k}\right] \\
& +h\left[-I^{e}+\left(X_{1, k}+G^{e}\right)^{(-0.6253)}\left(C_{1 g} H^{e}+C_{2 g} \dot{V}_{A}^{e}+C_{3 g}\right)\right],
\end{aligned}\right.
$$




$$
\begin{aligned}
& N L(X)=\left(X_{2}+I^{e}\right)^{(-1.3285)} \\
& \text { and } N L(X)=\left(X_{1}+G^{e}\right)^{(-0.6253)} .
\end{aligned}
$$

The objective is to linearize these terms. This mechanism allows determining the Takagi-Sugeno fuzzy system. For this, we apply the fuzzy strategy and we consider the case of health person who exercises most regularly by jogging. We take $G^{e}=90 \mathrm{mg} / \mathrm{dl}$ and $I^{e}=30$ $\mu \mathrm{U} / \mathrm{dl}$. The equilibrium of cardiovascular respiratory parameters values $H^{e}$ and $\dot{V}_{A}^{e}$ for someone who does physical activity are given by the table (See [17]).

We consider a universe of discourse $X$ which has two linguistic variables: glucose (GL) and insulin (INS). Taking account of the physiology, we consider $G \in[60,140]$ and $I \in[20,40]$. Therefore, the glucose (resp. insulin) is low if $G<60 \mathrm{mg} / \mathrm{dl}$ (resp. $I=<20$ $\mu \mathrm{U} / \mathrm{dl}$ ). If $G$ (resp. $I$ ) is included between 60 and $140 \mathrm{mg} / \mathrm{dl}$ (resp. 20 and $40 \mu \mathrm{U} / \mathrm{dl}$ ), we suppose that the glucose (resp. insulin) is normal. While if $G>140$ (resp. $I>40$ ) we say that the glucose (resp. insulin) is the highest. Then, GLB (Low glucose), GLN (Normal glucose) and GLE (the highest glucose) constitute the terms (fuzzy sets) of the linguistic variable GL. In an analogous way, INSB (Low insulin), INSN (Normal insulin) and INSE (the highest insulin) are the terms of the variable linguistic INS.

According the relation (18) and equilibrium values given by the Table 1, we have $X_{1} \in[-30,50]$ and $X_{2} \in[-15,5]$. During the physical activity, the glucose (resp. insulin) varies such that we can consider a universe of discourse $X$ where the labels are centered at $-30,10$ and 50 (resp. $-15,-5$ and 5 ). Then, we suppose that theses centers constitute the operating points values of the system (20). We designate these points as $M_{i}, \quad i=1,2,3$, for the first equation of the system (20) and as $N_{i}$, $i=1,2,3$, for the second. It is obvious that these points take the corresponding values in the labels centers of a universe of discourse $X$ [10]. Membership functions associated to this labeling are represented in the Figure 2.

To simplify, we consider only the Taylor expansion of first order around the operating points $M_{i}$ and $N_{i}$. We obtain three systems of the following form (see Equation (22))
Finally, the optimal control problem (1)-(2) can be formulated as follows.

Find $U^{*}=\left(U_{0}^{*}, \cdots, U_{N-1}^{*}\right)^{\mathrm{T}}$ solution of

$$
\min _{U} J(X, U)=\sum_{k=0}^{N-1}\left(X_{k}^{\mathrm{T}} R_{1} X_{k}+U_{k}^{\mathrm{T}} R_{2} U_{k}\right) h
$$

subject to

$$
X_{k+1}=A_{i, k} X_{k}+B_{i, k} U_{k}+C_{i, k}, \quad i=1,2,3,
$$

where

$$
\begin{gathered}
A_{i}=\left(\begin{array}{cc}
1-h & 0 \\
0 & 1-h
\end{array}\right), i=1,2,3 \\
B_{i}=h\left(\begin{array}{ll}
\left(N_{i}+I^{e}\right)^{(-0.6253)} \times C_{1 f} & \left(N_{i}+I^{e}\right)^{(-0.6253)} \times C_{2 f} \\
\left(M_{i}+G^{e}\right)^{(-1.3285)} \times C_{1 g} & \left(M_{i}+G^{e}\right)^{(-1.3285)} \times C_{2 g}
\end{array}\right), \\
i=1,2,3 \\
C_{i}=h\left(\begin{array}{l}
-G^{e}+\left(N_{i}+I^{e}\right)^{(-0.6253)}\left(C_{1 f} H^{e}+C_{2 f} \dot{V}_{A}^{e}+C_{3 f}\right) \\
-I^{e}+\left(M_{i}+G^{e}\right)^{(-1.3285)}\left(C_{1 g} H^{e}+C_{2 g} \dot{V}_{A}^{e}+C_{3 g}\right)
\end{array}\right), \\
i=1,2,3 .
\end{gathered}
$$

Let us set $N=100$ and $T_{\max }=10$, then the following results are found

$$
\begin{gathered}
A_{i}=\left(\begin{array}{cc}
0.9 & 0 \\
0 & 0.9
\end{array}\right), i=1,2,3 \\
B_{1}=\left(\begin{array}{cc}
0.2133 & 1.9578 \\
0.00011 & 0.0039
\end{array}\right), B_{2}=\left(\begin{array}{cc}
0.1550 & 1.4225 \\
0.00005 & 0.0019
\end{array}\right) \\
B_{3}=\left(\begin{array}{cc}
0.1256 & 1.1526 \\
0.00003 & 0.0013
\end{array}\right) \\
C_{1}=\left(\begin{array}{l}
-4.0142 \\
-2.9543
\end{array}\right), C_{2}=\left(\begin{array}{l}
-5.3775 \\
-2.9769
\end{array}\right), C_{3}=\left(\begin{array}{l}
-6.0648 \\
-2.9851
\end{array}\right) .
\end{gathered}
$$

Table 1. Equilibrium of cardiovascular-respiratory system parameters in the jogging case.

\begin{tabular}{ccccc}
\hline Parameter & $G^{e}(\mathrm{mg} / \mathrm{dl})$ & $I^{e}(\mu \mathrm{U} / \mathrm{dl}$ & $H^{e}($ Beats $/ \mathrm{min})$ & $\dot{V}_{A}^{e}(\mathrm{~L} / \mathrm{min})$ \\
\hline Value & 90 & 30 & 140 & 15 \\
\hline
\end{tabular}

$$
\begin{aligned}
X_{1, k+1}= & (1-h) X_{1, k}+h\left(N_{i}+G^{e}\right)^{(-1.3285)}\left[C_{1 f} \times U_{1, k}+C_{2 f} \times U_{2, k}\right] \\
& +h\left[-G^{e}+\left(N_{i}+G^{e}\right)^{(-1.3285)}\left(C_{1 f} H^{e}+C_{2 f} \dot{V}_{A}^{e}+C_{3 f}\right)\right], \\
X_{2, k+1}= & (1-h) X_{2, k}+h\left(M_{i}+I^{e}\right)^{(-0.6253)}\left[C_{1 g} U_{1, k}+C_{2 g} U_{2, k}\right] \\
& +h\left[-I^{e}+\left(M_{i}+I^{e}\right)^{(-0.6253)}\left(C_{1 g} H^{e}+C_{2 g} \dot{V}_{A}^{e}+C_{3 g}\right)\right] . \quad i=1,2,3
\end{aligned}
$$



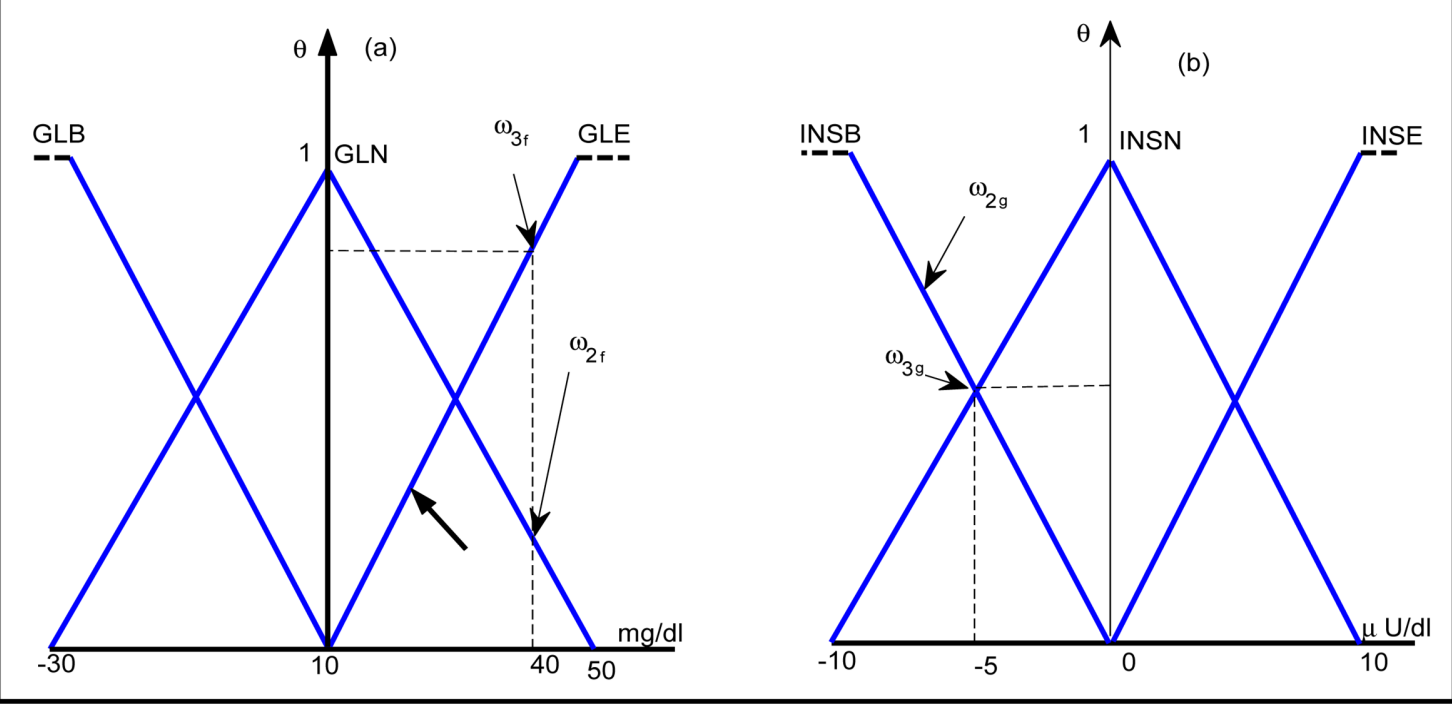

Figure 2. Triangular membership functions associated to operating points $-30,10$ and 50 (resp. $-10,0$ and 10) for the linguistic variable GL (a) (resp. INS (b)) according to the variable change (18). 40 and -5 are the values of entries obtained thanks to the formula (18).

It is easy to note that the problem (23)-(24) is a linear quadratic (LQ). Since there are three linear state systems, the solution leads to three feedback controls of the form

$$
U_{k}=-K_{i, k} X_{k}, \quad i=1,2,3 \text {; }
$$

where $K_{i}$ is a gain feedback.

The implementation can be made in several platforms. Here we use MATLAB package. If $R_{1}$ and $R_{2}$ are identified matrixes of the second order, we obtain

$$
\begin{aligned}
& K_{1}=\left(\begin{array}{cc}
1.1712 & -0.0081 \\
-0.0081 & 5.2631
\end{array}\right), K_{2}=\left(\begin{array}{cc}
1.2868 & -0.0055 \\
-0.0055 & 5.2631
\end{array}\right) \\
& K_{3}=\left(\begin{array}{cc}
1.3928 & -0.0042 \\
-0.0042 & 5.2632
\end{array}\right) .
\end{aligned}
$$

The defuzzification transformation allows to obtain one system. Consequently, for the system (23) this technique gives the following system

$$
X_{k+1}=A X_{k}+B U_{k}+C, k=0, \cdots, N-1,
$$

where $A$ and $B$ are $2 \times 2$ matrices and $C$ a $2 \times 1$ matrix.

In the same way, from the matrixes $K_{1}, K_{2}$, and $K_{3}$ the defuzzication process allows to have one matrix $K$. We propose the following procedure.

The first (second) line of matrixes $A_{i}, B_{i}, C_{i}$ and $K_{i}$, $i=1,2,3$, is defuzzified using the degree of membership $\omega_{2 f}$ and $\omega_{3 f}$ [see the Figure 2(a)] (resp. $\omega_{2 g}$ and $\omega_{3 g}$ [see the Figure 2(b)]). This manner of procedure is due to the two following reasons.

1) We consider the degree of membership of the entry glucose (resp. insulin). According to variable change (18), this value is $40 \mathrm{mg} / \mathrm{dl}$ [see the Figure 2(a)] (resp.
$-5 \mu \mathrm{U} / \mathrm{dl}$ [see the Figure 2(b)]). After calculations, we get $\omega_{1 f}=0, \quad \omega_{2 f}=0.25$ and $\omega_{3 f}=0.75 \quad\left(\theta_{G L}=(0\right.$; $0.25 ; 0.75))$ [resp. $\omega_{1 g}=0, \omega_{2 g}=0.5$ and $\omega_{3 g}=0.5$. $\left(\theta_{I N S}=(0 ; 0.5 ; 0.5)\right)$.

2) The nonlinear factor

$$
\begin{aligned}
& N L(X) \\
= & \left(X_{2}+I^{e}\right)^{(-1.3285)}\left(\operatorname{resp} . N L(X)=\left(X_{1}+G^{e}\right)^{(-0.6253)}\right)
\end{aligned}
$$

intervenes only in the first (resp. second) equation of the system (22).

Considering these hypothesis, we have the following matrixes.

$$
\begin{aligned}
& A=\left(\begin{array}{cc}
0.9 & 0 \\
0 & 0.9
\end{array}\right), \quad B=\left(\begin{array}{cc}
0.1462 & 1.3415 \\
0.00004 & 0.0015
\end{array}\right) \\
& C=\left(\begin{array}{l}
-5.5837 \\
-2.9831
\end{array}\right), \quad K=\left(\begin{array}{cc}
1.3186 & -0.00513 \\
-0.0046 & 5.2632
\end{array}\right) .
\end{aligned}
$$

\subsection{Direct Approach}

To approximate the system (2), let us consider

$$
\mathcal{B}^{N}=\left\{\psi_{j}^{N}, j=1, \cdots, N\right\}
$$

a linear B-splines basis functions on the uniform grid

$$
\Omega_{N}=\left\{t_{k}=\frac{k T_{\max }}{N}, k=0, \cdots, N\right\},
$$

such that

$$
\psi_{i}^{N}\left(t_{k}\right)=\delta_{i k}
$$

Let us introduce the vector space $W^{N}$ whose the ba- 
sis is $\mathcal{B}^{N}$. We have

- $\operatorname{dim} W^{N}=N$

- $W^{N} \subset W^{N+1}$

Let us consider $W=C^{0}(0, T)$ and let us take the interpolation operator

$$
\begin{aligned}
\Pi^{N}: W & \rightarrow W^{N} \\
\phi & \mapsto \Pi^{N} \phi
\end{aligned}
$$

satisfying

$$
\Pi^{N} \phi\left(t_{k}\right)=\phi\left(t_{k}\right), \quad k=1, \cdots, N .
$$

We verify easily that

$$
\begin{gathered}
\left\|\Pi^{N} \phi-\phi\right\|_{E} \underset{N \rightarrow \infty}{\longrightarrow} 0 \quad \forall \phi \in E \\
\left\|\Pi^{N}\right\| \|=\sup _{\substack{\phi \neq 0 \\
\phi \in W}} \frac{\left\|\Pi^{N} \phi\right\|_{E}}{\|\phi\|_{E}}=1 .
\end{gathered}
$$

Now, let us set

$$
f^{N}=\Pi^{N} f=\sum_{k=0}^{N} f^{k} \psi_{k}^{N}, \text { and } g^{N}=\Pi^{N} g=\sum_{k=0}^{N} g^{k} \psi_{k}^{N}
$$

with

$$
f^{k}=f\left(H\left(t_{k}\right), \dot{V}_{A}\left(t_{k}\right)\right), \text { and } g^{k}=g\left(H\left(t_{k}\right), \dot{V}_{A}\left(t_{k}\right)\right)
$$

Therefore, the system () can be approached by the following form

$$
\begin{aligned}
& \text { Find }\left(G^{N}, I^{N}\right) \in\left(W^{N}\right)^{2} \text { solution of the system } \\
& \begin{array}{c}
\frac{\mathrm{d} G^{N}}{\mathrm{~d} t}=-G^{N}+\left(I^{N}\right)^{(-1.3285)} f^{N} \\
\frac{\mathrm{d} P^{N}}{\mathrm{~d} t}=-I^{N}+\left(G^{N}\right)^{(-0.6253)} g^{N} \\
G^{N}(0)=P^{N, 0}, \quad I^{N}(0)=I^{N, 0},
\end{array}
\end{aligned}
$$

such that

$$
\begin{aligned}
& \left|G^{0}-G^{N, 0}\right| \underset{N \rightarrow \infty}{\longrightarrow} 0 \\
& \left|I^{0}-I^{N, 0}\right| \underset{N \rightarrow \infty}{\longrightarrow} 0 .
\end{aligned}
$$

According to this approximation, we have the following result.

Proposition [8]

The solution sequence of the system (34)-(36) converges uniformly toward the solution of the system (2) on the interval $\left[0, T_{\max }\right], T_{\max }>0$.

To approximate the optimal problem (1)-(2), let us set $x=(G, I)^{\mathrm{T}}$ the state vector, $x^{0}=\left(G^{0}, I^{0}\right)^{\mathrm{T}}$ the initial state vector, $x^{e}=\left(G^{e}, I^{e}\right)^{\mathrm{T}}$ the wanted equilibrium state vector, $\lambda=\left(H, \dot{V}_{A}\right)^{\mathrm{T}}$ the control vector and $\lambda^{e}=\left(H^{e}, \dot{V}_{A}^{e}\right)^{\mathrm{T}}$ the equilibrium control vector; $x_{i}, x_{i}^{0}, x_{i}^{e}, \lambda_{i}$ and $\lambda_{i}^{e}$ designate the $i^{\text {th }}$ components $i^{\text {th }}$ of the vector $x, x^{0}, x^{e}, \lambda$ and $\lambda^{e}$.

Therefore, the problem (1)-(2) can take the following compact form

$$
\begin{aligned}
& \min _{\lambda \in Q} J^{N}(\lambda) \\
= & \int_{0}^{T_{\max }}\left(\sum_{i=1}^{2} a_{i} \cdot\left(x_{i}^{N}(t)-x_{i}^{e}\right)^{2}+\sum_{j=1}^{2} b_{j} \cdot\left(\lambda_{j}(t)-\lambda_{j}^{e}\right)^{2}\right) \mathrm{d} t,
\end{aligned}
$$

where $x^{N}=\left(x_{1}^{N}, x_{2}^{N}\right)^{\mathrm{T}}$ is solution of the approximated solution (34)-(36).

We must determine $\lambda^{M}=\left(\lambda_{1}^{M}, \lambda_{2}^{M}\right) \in Q^{M}$ an approximate solution of (39) in $Q^{M}=\left(W^{M}\right)^{2}$. It is necessary to note that we can write

$$
\lambda_{j}^{M}=\sum_{k=0}^{M} \lambda_{j, k}^{M} \psi_{k}(t), j=1,2 .
$$

Therefore, we can approximate the objective function by

$$
\begin{aligned}
& J^{N}\left(\lambda^{M}\right) \\
\approx & \sum_{k=1}^{M}\left(\sum_{i=1}^{2} a_{i}\left(x_{i}^{N}\left(t_{k}\right)-x_{i}^{e}\right)^{2}+\sum_{j=1}^{2} b_{j}\left(\lambda_{j, k}^{M}-\lambda_{j}^{e}\right)^{2}\right) \Delta t,
\end{aligned}
$$

where $\Delta t=\frac{T_{\max }}{N}$. The convergence of the discreet objective function (41) toward the continuous objective function given by the problem (39) has been shown in [8].

Finally, the optimal control problem (1)-(2) is a minimisation problem with constraint. The discreet formulation of such problem can be written as follows.

Find $\lambda^{*, M} \in \mathbb{R}^{(M+1)} \times \mathbb{R}^{(M+1)}$ solution of

$$
\min _{\lambda^{M} \in \mathbb{R}^{(M+1)} \times \mathbb{R}^{(M+1)}} J^{N}\left(\underline{\lambda}^{M}\right) \approx \Delta t\left(\left(Y^{\mathrm{T}} R_{1} Y\right)+\left(\underline{\lambda}^{M}\right)^{\mathrm{T}} R_{2} \underline{\lambda}^{M}\right),
$$

subject to

$$
\left\{\begin{array}{l}
\frac{\mathrm{d} G^{N}}{\mathrm{~d} t}=-G^{N}+\left(I^{N}\right)^{(-1.3285)} f^{N} \\
\frac{\mathrm{d} I^{N}}{\mathrm{~d} t}=-I^{N}+\left(G^{N}\right)^{(-0.6253)} g^{N},
\end{array}\right.
$$

where $\underline{\lambda}^{M}$ is a matrix $(M+1) \times 2$ such that the components $\lambda_{j, k}^{M}$ are those function $\lambda_{j}^{N}$ in $\mathcal{B}^{N}$ and $Y$ is the matrix such that the $(i, k)^{\text {th }}$ component is $x_{i}^{N}\left(t_{k}\right)-x_{i}^{e}$, where $x^{N}=\left(x_{1}^{N}, x_{2}^{N}\right)^{\mathrm{T}}$ is the solution of (1)-(2) associated to $\lambda=\lambda^{N}$.

\section{Numerical Simulation}

We consider the acute cardiovascular respiratory response to glucose and insulin dynamic for a 30-year-old trained 
women whose mean values are given in Table 1 [17]. The solutions of the optimal control problem (23)-(24) and (42)-(43) can be determined in several platform. The implementation of these solutions is made using MATLAB packages.

To solve the problem (23)-(26) by fuzzy logic strategy only one program is enough. Using direct approach, the solutions of the problem (42)-(43) are given by a succession of programs based on MATLAB function used in optimization that is fmincon. This function is a MATLAB program which allows solving minimization problem with constraints.

In this section, we note by AHLF, ADIR to designate respectively the hybrid approach integrating fuzzy logic and direct approach. Consequently, the Table 2 gives us the results found after the execution of main MATLAB program for AHLF, ADIR respectively. The results are obtained using a Processor Intel(R) core (TM)2 Duo CPU, 2.20 GHZ.

Table 2 shows that the time execution of the main program to solve the problem (1)-(2) by AHLF is very small compared to one of ADIR. This argument justifies the precision of the fuzzy logic strategy.

Considering jogging as physical activity for a 30 -year old trained woman, the variations of the optimal parameters is obtained using the hybrid approach integrating fuzzy logic and the direct approach. The results are given in the curves represented in the Figure 3.

Table 2. Minimal values of the objective function $\left(J_{\text {opt }}\right)$ and the execution time $(T)$ of main program for the resolution of the optimal control problem (1)-(2) by AHLF, ADIR.

\begin{tabular}{ccc}
\hline & AHLF & ADIR \\
\hline$J_{\text {opt }}$ & 10.0524 & 35.8572 \\
$T$ (Second) & 5.8125 & 20.286 \\
\hline
\end{tabular}
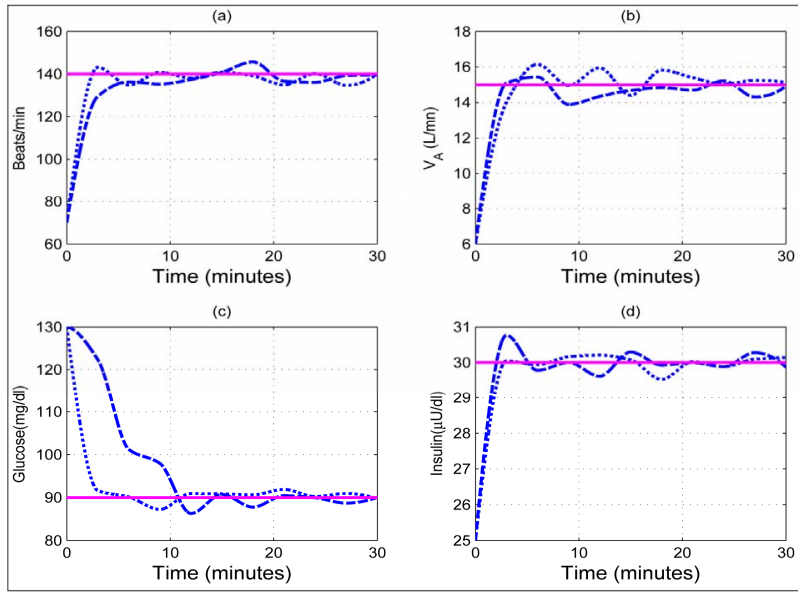

Figure 3. Optimal parameters for a 30 year old woman with jogging as her physical activity. The curve in solid line represents the wanted value. The curve in dashed line indicates the optimal parameter for the hybrid approach integrating the fuzzy logic strategy.
For a 30-year-old woman, the heart rate and alveolar ventilation play a crucial role in the control of the cardiovascular-respiratory system. Consequently, their stability ensures the performance of sportsman in general and of woman in particular. For a woman of 30 years old where jogging is her regular physical activity, we see that 2.5 minutes after the starting of the exercise the variation of the heart rate reaches the value close to the equilibrium value before having its oscillation around this value (Figure 3(a)). The solutions from AHLF and ADIR show that after 5 minutes of the starting time the optimal alveolar ventilation (Figure 3(b)) increases to reach the wanted equilibrium value and the glucose (Figure 3(c)) decreases gradually to reach the wanted value in 10 minutes before its oscillation around this value. The use of these approaches allows also the optimal insulin to increase and to reach the maximum that is close to the wanted value at $3^{\text {th }}$ minute before its oscillation around his value (Figure 3(d)). Comparing the results using AHLF and ADIR, it is important to see in the Figure 3 that they are much closed.

\section{Conclusion Remarks}

In this work, we compared two approaches to determine the optimal trajectories of glucose and insulin as response to controls of cardiovascular-respiratory system subjected to a physical activity. The finding results for two used methods are satisfactory and closed. But the hybrid approach integrating the fuzzy logic strategy has an advantage over the direct approach in term of time. Consequently, it constitutes an important approach for the resolution of the optimal control problem. In particular, it gives the optimal trajectories of glucose-insulin system in the same way so thet it ensures their performance.

\section{REFERENCES}

[1] R. N. Bergman, D. T. Finegood and S. E. Kahn, "The Evolution of Beta-Cell Dysfunction and Insulin Resistance in Type 2 Diabetes," European Journal of Clinical Investigation, Vol. 32, Suppl. 3, 2002, pp. 35-45. http://dx.doi.org/10.1046/j.1365-2362.32.s3.5.x

[2] V. W. Bolie, "Coefficients of Normal Blood Glucose Regulation," Journal of Applied Physiology, Vol. 16, No. 5, 1961, pp. 783-788.

[3] R. N. Bergman, L. S. Phillips and C. Cobelli, "Physiologic Evaluation of Factors Controlling Glucose Tolerance in Man: Measurement of Insulin Sensitivity and Beta-Cell Glucose Sensitivity from the Response to Intravenous Glucose," The Journal of Clinical Investigation, Vol. 68, No. 6, 1981, pp. 1456-1467. http://dx.doi.org/10.1172/JCI110398

[4] C. Cobelli, G. Pacini, G. Toffolo and L. Sacca, "Estimation of Insulin Sensitivity and Glucose Clearance from Minimal Model: New Insights from Labeled IVGTT," Ame- 
rican Journal of Physiology, Vol. 250, No. 5, 1986, pp. E591-E598.

[5] C. Cobelli, A. Caumo and M. Omenetto, "Minimal Model SG Overestimation and SI Underestimation: Improved Accuracy by a Bayesian Two-Compartment Model," American Journal of Physiology, Vol. 277, No. 3, 1999, pp. E481-E488.

[6] R. Hovorka, F. Shojaee-Moradie, P. V. Carroll, L. J. Chassin, I. J. Gowrie, N. C. Jackson, R. S. Tudor, A. M. Umpleby and R. H. Jones, "Partitioning Glucose Distribution/Transport, Disposal, and Endogenous Production during IVGTT," American Journal of Physiology: Endocrinology and Metabolism, Vol. 282, No. 5, 2002, pp. E992-E1007.

[7] M. S. Anirban Roy and R. S. Parker, "Dynamic Modeling of Exercise Effects on Plasma Glucose and Insulin Levels," Journal of Diabetes Science and Technology, Vol. 1, No. 3, 2007, pp. 338-347.

[8] J. M. Ntaganda and B. Mampassi, "Modelling Glucose and Insulin in Diabetic Human during Physical Activity," 2012 Proceeding of the 4th International Conference on Mathamatical Sciences (ICM), United Arab Emirates University, 11-14 March 2012, pp. 331-344.

[9] S. J. Yakowitz, "The Stagewise Kuhn-Tucker Condition and Differential Dynamic Programming," IEEE Transactions on Automatic Control, Vol. 31, No. 1, 1986, pp. 2530.http://dx.doi.org/10.1109/TAC.1986.1104123

[10] N. K. Masmoudi, C. Rekik, M. Djemel and N. Derbel, "Decomposition and Hierarchical Control for Discrete
Complex Systems by Fuzzy Logic Controllers," SSD '09. 6th International Multi-Conference on Systems, Signals and Devices, Djerba, 23-26 March 2009, pp. 1-6.

[11] M. Sugeno and K. Murakami, "Fuzzy Parking Control of Model Car," The 23rd IEEE Conference on Decision and Control, Vol. 23, 1984, pp. 902-903.

[12] T. Takagi and M. Sugeno, "Fuzzy Identification of Systems and Its Applications to Modeling and Control," IEEE Transactions on Systems, Man and Cybernetics, Vol. 15, No. 1, 1985, pp. 116-132.

http://dx.doi.org/10.1109/TSMC.1985.6313399

[13] M. I. Park, E. Kim, S. Ji and M. Park, "A New Approach to Fuzzy Modeling," IEEE Transactions on Fuzzy Systems, Vol. 5, No. 3, 1987, pp. 328-337.

[14] D. Jacobson, D. Lele and J. L. Speyer, "New Necessary Conditions of Optimality for Control Problems with State-Variable Inequality Constraints," Journal of Mathematical Analysis and Applications, Vol. 35, No. 2, 1971, pp. 255-284. http://dx.doi.org/10.1016/0022-247X(71)90219-8

[15] H. Kwakernaak and R. Savan, "Linear Optimal Control Systems," Wiley Inter-Science, New York, 1972.

[16] E. Trélat, "Contrôle Optimal: Théorie et Applications," Vuibert, Collection Mathématiques Concrètes, Paris, 2005.

[17] J. M. Ntaganda, B. Mampassi and D. Seck, "Modelling Blood Partial Pressures of the Human Cardiovascular/ Respiratory System," Applied Mathematics and Computation, Vol. 187, No. 2, 2007, pp. 1100-1108. http://dx.doi.org/10.1016/j.amc.2006.09.016 